\title{
3D-Printed Linear Positioner with Micrometer Accuracy
}

\author{
Yin-Yen Kuo, Chiao-Hua Cheng and Shao-Kang Hung \\ National Chiao Tung University, Department of Mechanical Engineering, 30010 Hsinchu City, Taiwan
}

\begin{abstract}
This article presents a positioner, whose flexure main body is made by a commercial 3D printer. Using this method, manufacturing a positioner can be cost efficient and much easier to customize. Integrating a laser displacement sensor, an electromagnetic actuator, and a feedback controller, this positioning system has 100 micron translational stroke with 1 micron resolution. Experiments also demonstrate sinusoidal motions at different frequencies. Using the method developed by this article, micro-positioners with customized specifications can be implemented rapidly, iteratively, and cost-effectively.
\end{abstract}

\section{Introduction}

Precision positioners are typical mechatronic devices which are widely used in automatic manufacturing [1], [2] and measurement. In the field of atomic level topography, they can be equipped in scanning tunneling microscopes [3], [4] and atomic force microscope [5]-[7]. Using piezoelectric elements in devices for positioning is a common method to achieve the goal of precision [8]. The advantages of piezoelectric are light-weight, high resolution, and fast response. Despite of all the advantages mentioned, a crucial shortcoming, short traveling range, will limit the applications. To find the solution of long traveling range, there are many researches of piezoelectric positioning [9]. One of the positioning solutions is apply electromagnetic actuators, which do not need to contact mechanisms and possess the ability to achieve a larger displacement.

The cooperation of electromagnetic and flexure hinge mechanism becomes popular nowadays. Utilizing the flexure hinge mechanism [8] has many advantages, such as no backlash, smoothly continuous displacement and no friction between mechanisms. Nevertheless, most of the flexure hinge mechanisms are manufactured by electrical discharge machining (EDM) process [10]-[12] with metal materials, which often spends lots of effort and cost. In order to improve the shortcomings, we decided to manufacture flexure hinge mechanism by 3D-printing [13] with polylactic acid (PLA) material. In this case, not only the cost of producing is reduced, but also making it easier to implement or to be improved iteratively.

In this paper, a 3D-printed flexure hinge positioner cooperate with electromagnetic actuator is presented. Moreover, experiment results reveal the satisfactory performance.

\section{System design}

Due to the rapid development of 3D-printing, also known as additive manufacturing, we can customize many complicated structures easily [14], [15]. Fig. 1(a) shows the top view of the experimental setup including a permanent magnet, a coil, a moving stage, and a laser displacement sensor.

(a)

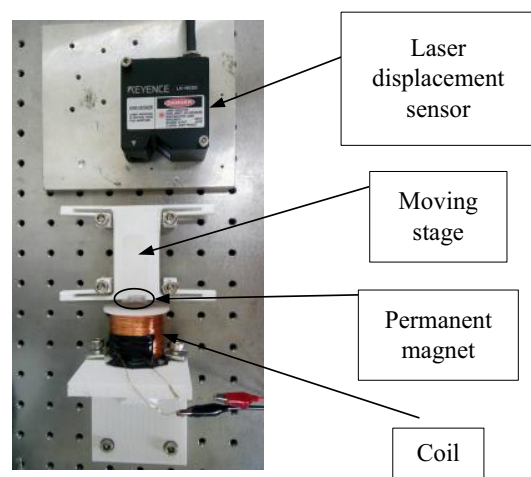

(b)

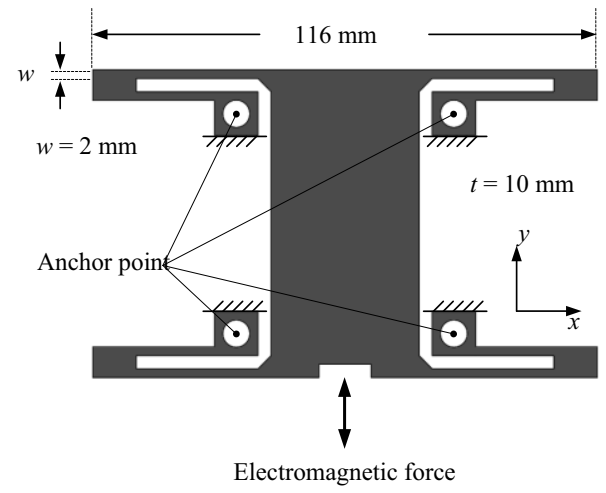

Figure 1. (a) The experimental setup and (b) the detailed structure of moving stage. 
A permanent magnet is fixed at one end of the moving stage for interacting with the coil. A laser displacement sensor (LK-H020, KEYENCE) is fixed facing the other end of the moving stage for measuring displacement. The detailed design of the moving stage is presented in Fig. 1(b). The thickness $t$ of the moving stage is $10 \mathrm{~mm}$. The width $w$ is designed to $2 \mathrm{~mm}$ because of the limitation of printing quality of the commercial 3D printer. With the flexure structure, the moving stage can move smoothly in $y$ direction.

Fig. 2 shows the schematic block diagram. For the purpose of achieving high-precision positioning, an appropriate feedback controller is necessary. Using proportion-integral-derivative control scheme is a common method, and it can be easily applied in many systems. However, in this paper, a proportion-integral (PI) control scheme is enough. We utilize PI closed-loop control to overcome the hysteresis problem and reduce the steady stare error of the mechanical motion. Furthermore, an optical table is used to stabilize the whole system in order to decrease vibration from external environment.

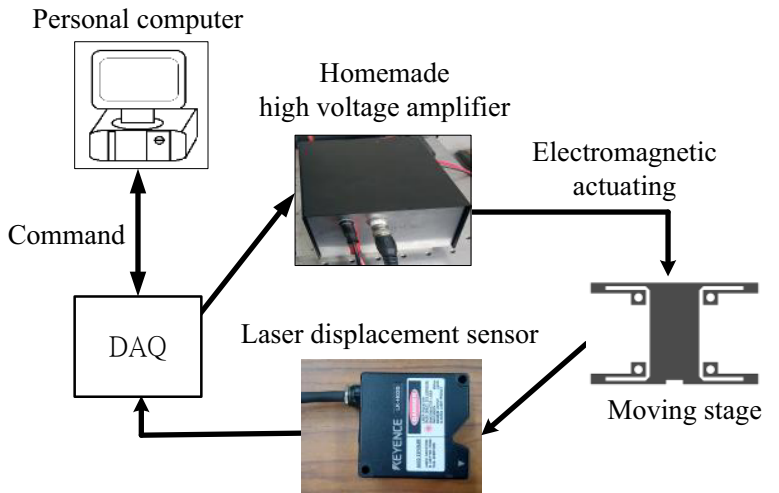

Figure 2. The block diagram of the proposed design.

National Instrument USB-6341 Data Acquisition (DAQ) card is selected to integrate the whole system. Also, a homemade high voltage amplifier is used to magnify the output voltage of the DAQ signal by 10 times. The maximum range can reach from $-30 \mathrm{~V}$ to +30 $\mathrm{V}$.

In order to prevent from the electromagnetic interference, the contactless optical sensor is chosen in the experiments. Having the active range from $-3 \mathrm{~mm}$ to $+3 \mathrm{~mm}$ and $10 \mathrm{k} \mathrm{Hz}$ sampling frequency, the laser displacement sensor is utilized as the feedback sensor. The optical sensor has another advantage of avoiding the impact of the unevenness surface, which is especially significant in the 3D-printed surface relative to the EDM metal surface.

The 3D printer used in this article is AURORA 3D printer F1, shown in Fig. 3. The product specification of AURORA 3D printer F1 is indicated in Table 1. The build envelope is $168 \mathrm{~mm} \times 168 \mathrm{~mm} \times 168 \mathrm{~mm}$, which is sufficient to print the moving stage in this article. PLA is biodegradable, and has lower melting point compared to Acrylonitrile Butadiene Styrene (ABS). Also, Unlike ABS, PLA is possible to print successfully without heating the printing bed first. Moreover, When PLA is heated, it will become much more liquid, which leads to demonstrate much sharper details. With all the advantages mentioned, the AURORA 3D printer F1 and PLA material are chosen.

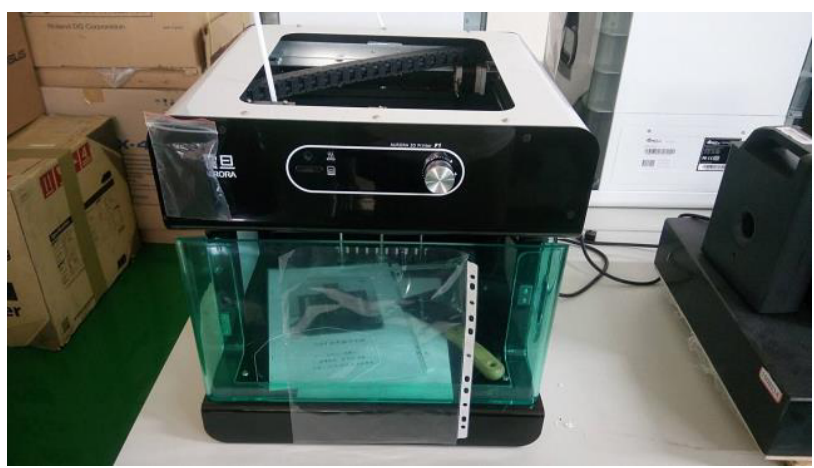

Figure 3. AURORA 3D printer F1

Table 1. AURORA 3D printer F1 product specifications

\begin{tabular}{|c|c|}
\hline Overall dimensions & $450 \mathrm{~mm}^{*} 420 \mathrm{~mm} * 460 \mathrm{~mm}$ \\
\hline Weight & $19 \mathrm{~kg}$ \\
\hline Build envelope & $168 \mathrm{~mm}^{*} 168 \mathrm{~mm} * 168 \mathrm{~mm}$ \\
\hline $\begin{array}{c}\text { Minimum } \\
\text { layer thickness }\end{array}$ & $0.1 \mathrm{~mm}$ \\
\hline Material & PLA $\varnothing 1.75 \mathrm{~mm} \pm 0.1 \mathrm{~mm}$ \\
\hline Nozzle diameter & $0.4 \mathrm{~mm}$ \\
\hline
\end{tabular}

\section{Experiment result}

\subsection{Large step response}

The full stroke capability of the proposed design mentioned in this paper can achieve from $-50 \mu \mathrm{m}$ to $+50 \mu \mathrm{m}$. Fig. 4 shows the large step response ranged from $0 \mu \mathrm{m}$ to $50 \mu \mathrm{m}$. In the configuration, we can see that the overshoot is about $3 \%$ and the settling time is about 0.3 second.

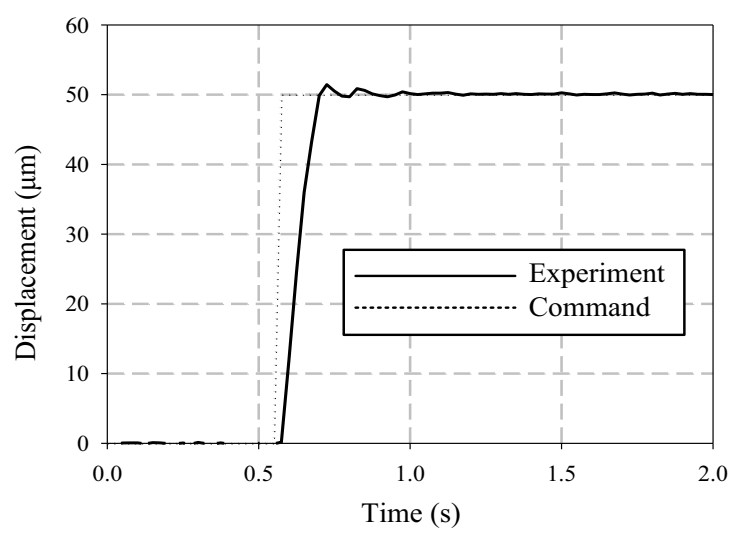

Figure 4. The large step response.

\subsection{Step-train response}

While Step-train motion is one of the most common operations of the positioning system, we make some experiments of continuing stepping response. In Fig. 5(a), the moving stage moves between $-50 \mu \mathrm{m}$ and $+50 \mu \mathrm{m}$ with each step equal to $5 \mu \mathrm{m}$. And in Fig. 5(b), the 
moving stage moves between $-5 \mu \mathrm{m}$ and $+5 \mu \mathrm{m}$ with each step equal to $1 \mu \mathrm{m}$.

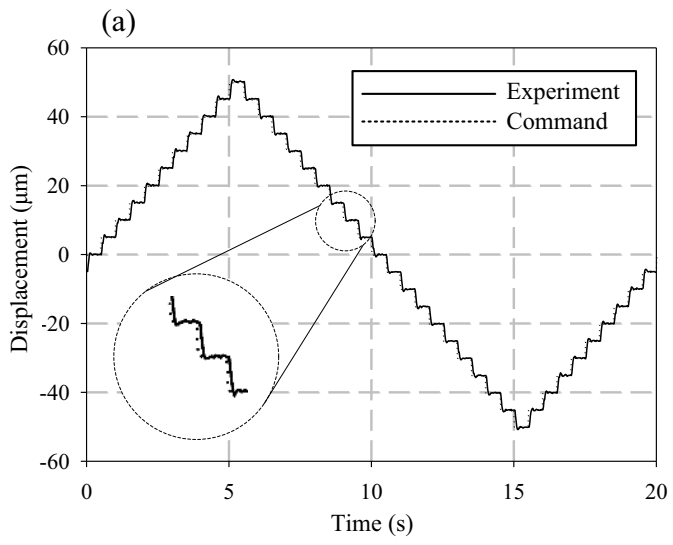

(b)

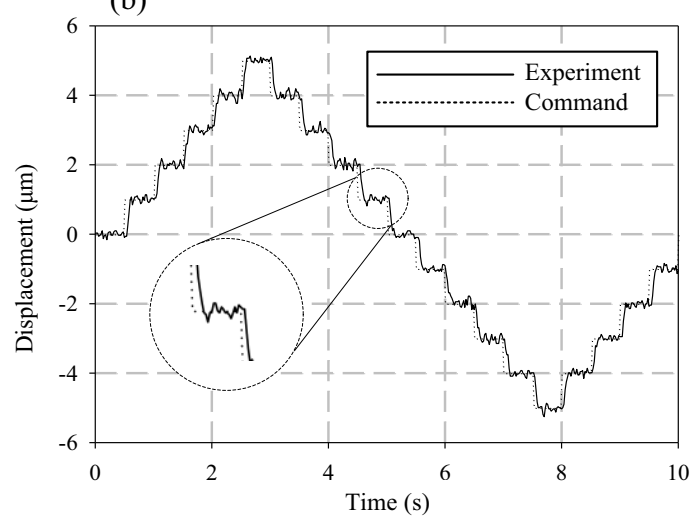

Figure 5. The step-train motion with range (a) $\pm 50 \mu \mathrm{m}$ with 5 $\mu \mathrm{m}$ each step and (b) $\pm 5 \mu \mathrm{m}$ with $1 \mu \mathrm{m}$ each step.
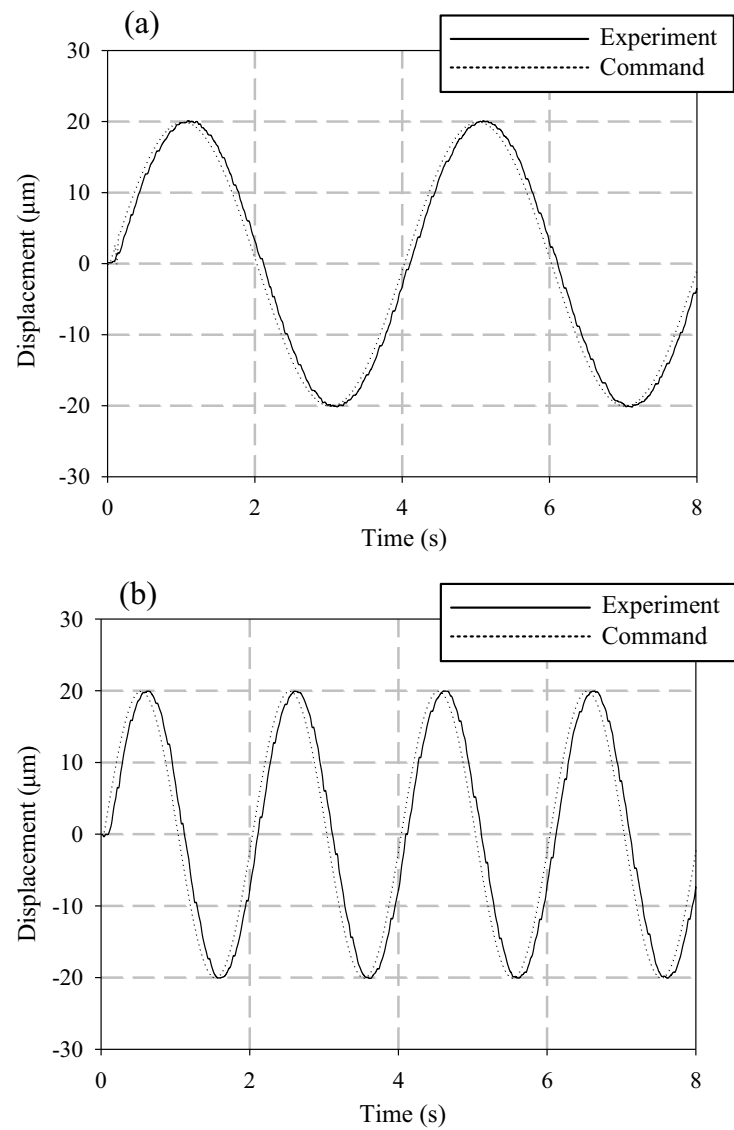

(c)

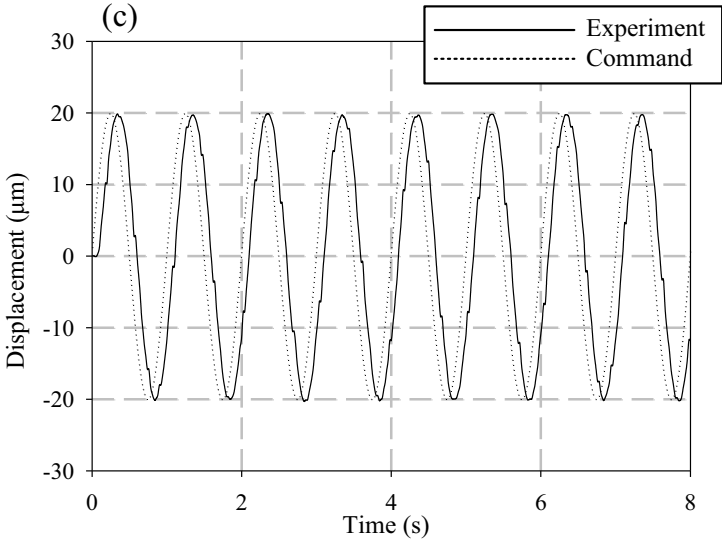

(d)

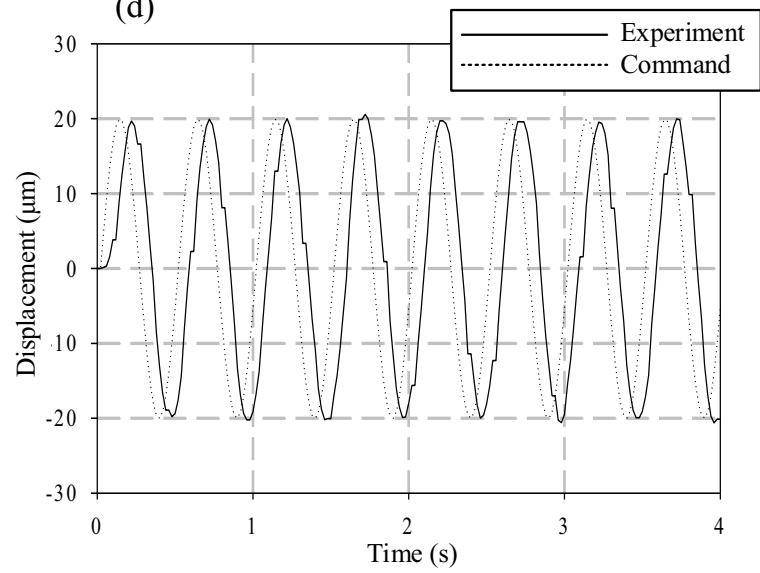

Figure 6. The sinusoidal motion, (a) $0.25 \mathrm{~Hz}$, (b) $0.5 \mathrm{~Hz}$, (c) 1 $\mathrm{Hz}$ and (d) $2 \mathrm{~Hz}$.

\subsection{Sinusoidal response}

The result of the sinusoidal response is illustrated for testing the tracking capability of the proposed design. With the amplitude of $20 \mu \mathrm{m}$, the frequency at $0.25 \mathrm{~Hz}$, $0.5 \mathrm{~Hz}, 1 \mathrm{~Hz}$ and $2 \mathrm{~Hz}$ are presented in Fig. 6(a)-(d), respectively. As the frequency exceeds $1 \mathrm{~Hz}$, the performance becomes unsatisfactory.

\section{Conclusion and outlook}

A novel approach of manufacturing micro-positioner is demonstrated in this article. With 3D-printing technique, fabricating customized positioners costs less effort, time, and budget. The proposed design has $100 \mu \mathrm{m}$ traveling, 1 $\mu \mathrm{m}$ resolution, and sinusoidal motion tracking ability. This design will be soon improved iteratively in the future. More advanced control schemes are planned to enhance the dynamical performance of our next generation 3D-printed positioner.

\section{References}

1. T. Hu, W. J. Kim, IEEE/ASME Trans. Mech., 11, 682 (2006)

2. I. J. Busch-Vishniac, IEEE Circuits Devices Mag. 7, 32 (1991) 
3. G. Binnig, H. Rohrer, Surf. Sci., 126, 236-244(1983)

4. K. Uozumi, K. Nakamoto, K. Fujioka, Jpn. J. Appl. Phys., 27, L123-L126 (1988)

5. G. Binnig, C. F. Quate, Ch. Gerber, Phys. Rev. Lett., 56, 9 (1986)

6. S. K. Hung, L. C. Fu, IEEE Trans. Instrum. Meas., 55, 5 (2006)

7. S. Hwang, S. K. Hung, L.C. Fu, M. Y. Lin - US Patent 7249494 (2007)

8. H. R. Lin, C. H. Cheng, S. K. Hung, Mechatronics, 31, 180-188 (2015)

9. W. Dong, L. N. Sun, Z. J. Du, Sens. Actuator APhys., 135, 250-256 (2007)
10. E. C. Jameson, Electrical Discharge Machining (Society of Manufacturing Engineers, 2001)

11. Y. C. Lin, C. C. Tsao, C. Y. Hsu, S. K. Hung, D.C. Wen, Int. J. Adv. Manuf. Technol., 62, 1013-1023 (2012)

12. M. Y. Chen, H. H. Huang, S. K. Hung, IEEE Trans. Ind. Electron., 57, 1 (2010)

13. I. Gibson, D. Rosen, B. Stucker, Additive Manufacturing Technologies (Springer, 2010)

14. R. R. Ma, LU. Odhner, A. M. Dollar, Int. Conf. Robot. Autom., 2737-2743 (2013)

15. R. MacCurdy, R. Katzschmann, Y. Kim, D. Rus, 2016 IEEE Int. Conf. Robot. Autom., 3878-3885 (2016) 